\title{
Estimation of synchrotron-radiation background based on a real beam orbit
}

\author{
Tetsuo Abe* \\ Accelerator Laboratory, High Energy Accelerator Research Organization (KEK), Tsukuba, Ibaraki 305-0801, Japan
}

Hitoshi Yamamoto

Research Center for Neutrino Science (RCNS), Tohoku University, Sendai, Miyagi 980-8578, Japan

(Received 8 April 2004; published 23 July 2004)

\begin{abstract}
Some high-energy experiments have suffered from bitter experience on synchrotron-radiation background. As a measure, we have developed a new calculation method of synchrotron radiation based on a real beam orbit, aiming at quantitative estimations of synchrotron-radiation backgrounds, and the construction of a possible alarm system for the background. An explanation and a demonstration of our method are given.
\end{abstract}

DOI: 10.1103/PhysRevSTAB.7.072802

PACS numbers: 29.20.Dh, 29.27.Fh

\section{INTRODUCTION}

At the initial stage of the KEK B-factory (KEKB) experiment [1], synchrotron radiation (SR) from the circulating $8 \mathrm{GeV}$ electron beam caused serious damage to the Belle silicon vertex detector (SVD) [2], which was located close to the interaction point (IP). Some of the preamplifiers mounted on the innermost SVD layer died in about 10 days just after start of the gain-drop. Other high-energy experiments have also suffered from SR background.

We have developed a new method to calculate SR wattages based on a real beam orbit, aiming at quantitative estimations and the construction of a possible alarm system for SR background. Here, the real orbit is obtained by fitting measurements of beam-position monitors (BPMs). In the following sections, our method is explained together with some illustrations using the $8 \mathrm{GeV}$ electron beams of the KEKB High Energy Ring (HER). Finally, we present a simulation of the orbit and the gain-drop at the time of the SVD gain-drop accident as a benchmark.

\section{ORBIT CALCULATION}

Beam orbits are calculated by a linear approximation using transfer matrices, which are shown in the Appendix. For numerical calculations, we coded a dedicated computer program in FORTRAN, where the correctness of the orbit calculation was checked by comparing with a calculation using SAD [3]. Furthermore, we confirmed that there was no significant effect from round-off errors by comparing the results of double- and quadrupleprecision calculations.

*Email address: tetsuo.abe@kek.jp

\section{A. Fitting procedure}

Orbit fitting is performed based on the optics, field strengths of the magnets, and BPM measurements. We require minimization of $\chi^{2}$, defined as

$\chi^{2}=\sum_{j: \mathrm{BPM}}\left\{\left(X_{j}^{(\mathrm{BPM})}-X_{j}^{(\text {orbit })}\right)^{2}+\left(Y_{j}^{(\mathrm{BPM})}-Y_{j}^{(\text {orbit })}\right)^{2}\right\} / \sigma_{j}^{2}$,

where $X_{j}^{(\mathrm{BPM})}\left(Y_{j}^{(\mathrm{BPM})}\right)$ indicates the horizontal (vertical) beam position measured with BPM:j, $X_{j}^{\text {(orbit) }}\left(Y_{j}^{\text {(orbit) }}\right)$ the horizontal (vertical) position of the calculated orbit at BPM: $j$, and $\sigma_{j}$ is the BPM: $j$ resolution. There are four floating parameters, which are the orbit positions at the entrance and exit of the relevant accelerator section: $X^{(\mathrm{in})}, Y^{(\mathrm{in})}, X^{(\mathrm{out})}, Y^{(\mathrm{out})}$. Minimization of $\chi^{2}$ is performed using the computer program MINUIT [4,5]. An example of the orbit-fitting results for KEKB HER is shown in Fig. 1. Here, we define the vertical and horizontal $\chi^{2}$ to estimate the goodness of the fit for each direction separately:

$$
\begin{aligned}
& \chi_{X}^{2}=\sum_{j: \mathrm{BPM}}\left(X_{j}^{(\mathrm{BPM})}-X_{j}^{(\text {orbit })}\right)^{2} / \sigma_{j}^{2}, \\
& \chi_{Y}^{2}=\sum_{j: \mathrm{BPM}}\left(Y_{j}^{(\mathrm{BPM})}-Y_{j}^{(\text {orbit })}\right)^{2} / \sigma_{j}^{2},
\end{aligned}
$$

which lead to the following relations:

$$
\begin{gathered}
\chi^{2}=\chi_{X}^{2}+\chi_{Y}^{2}, \\
\frac{\chi^{2}}{N_{d f}}=\frac{\left[\left(\chi_{X}^{2} / N_{d f(X)}\right)\left(1 / N_{d f(Y)}\right)\right]+\left[\left(\chi_{Y}^{2} / N_{d f(Y)}\right)\left(1 / N_{d f(X)}\right)\right]}{\left(1 / N_{d f(X)}\right)+\left(1 / N_{d f(Y)}\right)},
\end{gathered}
$$

where $N_{d f(X)}\left(N_{d f(Y)}\right)$ indicates the number of degrees of freedom related to the horizontal (vertical) direction, and $N_{d f}$ for both: $N_{d f}=N_{d f(X)}+N_{d f(Y)}$. In the example shown in Fig. 1, the goodness of the fit is 

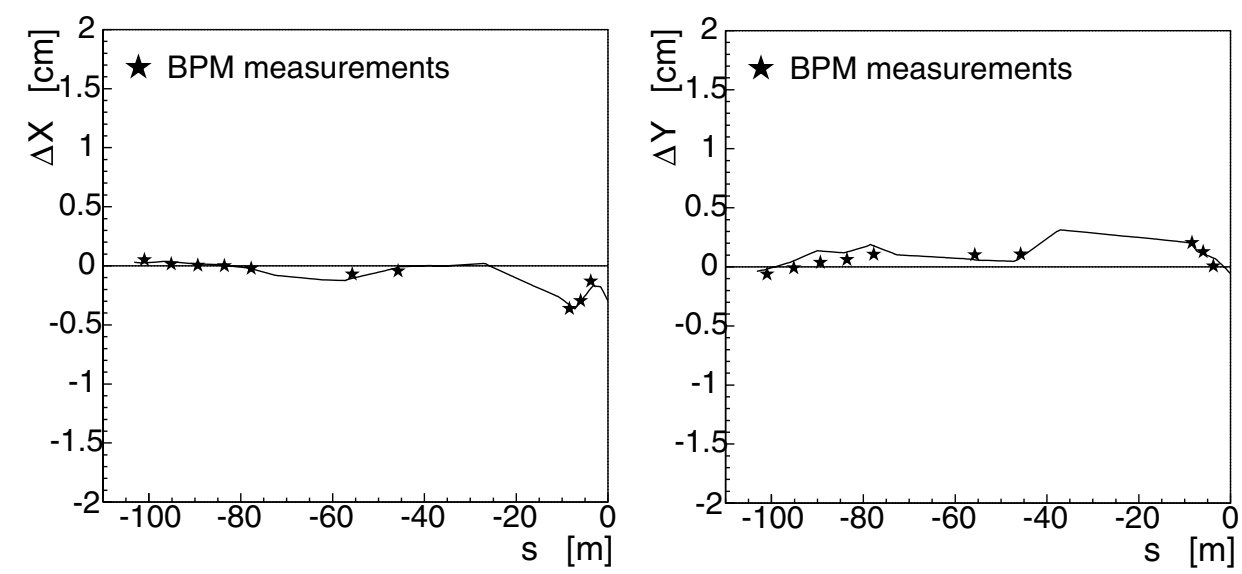

FIG. 1. Example of the orbit-fitting results for KEKB HER around the IP ( $s=\Delta X=\Delta Y=0)$ without any correction. The left (right) figure shows the horizontal (vertical) fitted orbit together with the BPM measurements. The $s$ axis is taken to be along the orbit. The horizontal line with $\Delta X=\Delta Y=0$ corresponds to the design orbit. The HER beam flows from the left to the right. $\chi_{X}^{2} / N_{d f(X)}=10.5$ and $\chi_{Y}^{2} / N_{d f(Y)}=40.3$.

$\chi_{X}^{2} / N_{d f(X)}=10.5$ and $\chi_{Y}^{2} / N_{d f(Y)}=40.3$ for a conservative BPM resolution of $100 \mu \mathrm{m}$. As is often the case, $\chi^{2}$ is too large, and also the orbit at the IP is unrealistically deflected in the negative direction by about $3 \mathrm{~mm}$. This is due to the fact that the absolute positions of magnets and BPMs are not known sufficiently well, leading to a need for the offset corrections.

\section{B. Offset corrections}

We modify the $\chi^{2}$ formula (1) as follows:

$$
\begin{aligned}
\chi^{2}= & \sum_{i: \text { orbit }} \sum_{j: \mathrm{BPM}}\left\{\left[\left(X_{j, i}^{(\mathrm{BPM})}+\Delta X_{j}^{(\mathrm{QUAD})}+\Delta X_{j}^{(\mathrm{BPM})}\right)\right.\right. \\
& \left.-X_{j, i}^{(\mathrm{orbit})}\right]^{2}+\left[\left(Y_{j, i}^{(\mathrm{BPM})}+\Delta Y_{j}^{(\mathrm{QUAD})}+\Delta Y_{j}^{(\mathrm{BPM})}\right)\right. \\
& \left.\left.-Y_{j, i}^{\text {(orbit) }}\right]^{2}\right\} / \sigma_{j}^{2},
\end{aligned}
$$

where $\Delta X_{j}^{(\mathrm{QUAD})}\left(\Delta Y_{j}^{(\mathrm{QUAD})}\right)$ indicates the horizontal (vertical) offset, with respect to the design position, of the real field center of the quadrupole magnet (QUAD) on which BPM: $j$ is mounted, and $\Delta X_{j}^{(\mathrm{BPM})}\left(\Delta Y_{j}^{(\mathrm{BPM})}\right)$ the horizontal (vertical) offset of the real origin of the BPM: $j$ measurement with respect to the real field center of QUAD:j. The index $i$ runs for different orbits in the case of a global fitting, which is explained in the next paragraph.

In order to obtain the offset sizes $\left(\Delta X_{j}^{(\mathrm{QUAD})}, \Delta Y_{j}^{(\mathrm{QUAD})}\right.$, $\Delta X_{j}^{(\mathrm{BPM})}$, and $\left.\Delta Y_{j}^{(\mathrm{BPM})}\right)$, we perform a global fitting for different orbits within a time period in which magnets are not expected to have moved. In the case of KEKB, we make various measurements in machine studies using six different orbits in each of the horizontal and vertical directions, which are produced by making a small kick at one of the six steering magnets. We fit those six orbits simultaneously with not only the usual floating parameters $\left[X_{i}^{\text {(in) }}, Y_{i}^{(\text {in })}, X_{i}^{\text {(out) }}, Y_{i}^{\text {(out) }}(i=1,2,3,4,5,6)\right]$, but also the offsets in formula (6). We furthermore introduce an additional floating parameter: the correction factor of the field strength of the bending magnet (BEND), $a$, which is defined as $\theta_{\text {corr }}=(1+a) \theta_{\text {org }}$, where $\theta_{\text {org }}$ and $\theta_{\text {corr }}$ are the kick angles before and after the correction, respectively. The BPM and QUAD offsets as well as the correction factors of the BENDs are common among the six orbits. It is clear that the simple fitting procedure leads to failure because there are too many floating parameters (more than 100 in our example).

Therefore, we first categorize the offset and correctionfactor parameters to be floating into three sets: the BPMrelated, QUAD-related, and BEND-related ones. We choose zero or one parameter to be floating in each category, and the other parameters are fixed at zero. Then, a global fitting is performed for the combination of the choice. We try all of the possible combinations (about $10 \times 10^{6}$ in our example), and a large number of results are filtered, requiring the following criteria: (i) the minimization of $\chi^{2}$ converges with a positivedefinite Hessian matrix, (ii) $\left|\Delta X_{j}^{(\mathrm{QUAD})}\right|<10 \mathrm{~mm}$ and $\left|\Delta Y_{j}^{(\mathrm{QUAD})}\right|<10 \mathrm{~mm}, \quad$ (iii) $\left|\Delta X_{j}^{(\mathrm{BPM})}\right|<10 \mathrm{~mm} \quad$ and $\left|\Delta Y_{j}^{(\mathrm{BPM})}\right|<10 \mathrm{~mm}$, (iv) $|a|<0.2$, (v) the vertical and horizontal displacements of the fitted orbit at the IP with respect to the nominal IP are within $1 \mathrm{~mm}$.

Then, the best (lowest) $\chi^{2}$ sample is selected as the final one. Figure 2 shows the fitted orbit after the offset corrections. With the corrections, the $\chi^{2}$ is small, and the orbit passes close to the BPM measurements and the nominal IP. Choosing two offsets to be floating in each category, we obtained almost the same results.

This method is to find a solution with a minimum modification within the scope of the numerical approach. 

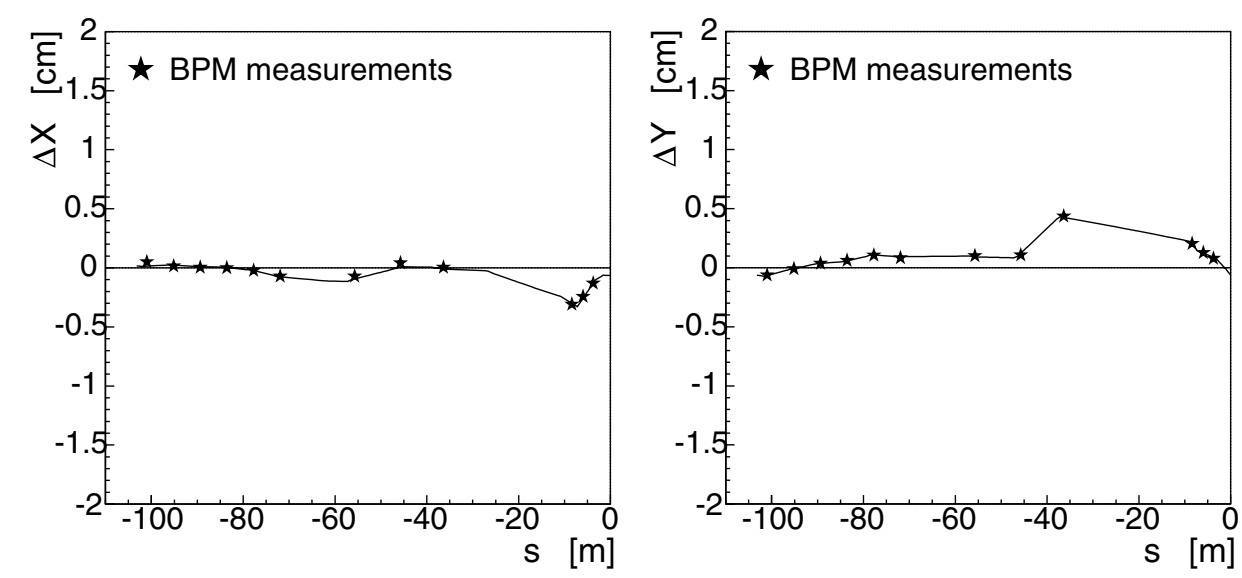

FIG. 2. Example of the orbit-fitting results after the offset corrections. The left (right) figure shows the horizontal (vertical) fitted orbit together with the BPM measurements. $\chi_{X}^{2} / N_{d f(X)}=3.6$ and $\chi_{Y}^{2} / N_{d f(Y)}=0.6$.

\section{WATTAGE CALCULATION}

SR wattages are calculated, based on the fitted real orbit, using the following analytical formula [6]:

$$
\begin{aligned}
\Delta^{3} W= & \frac{3 \alpha}{4 \pi^{2}} \frac{I}{e} \gamma^{2} \Delta \omega \Delta \phi \Delta \psi \frac{1}{\omega}\left(\frac{\omega}{\omega_{c}}\right)^{2}\left(1+\xi^{2}\right)^{2} \\
& \times\left\{K_{2 / 3}(\eta)^{2}+\frac{\xi^{2}}{1+\xi^{2}} K_{1 / 3}(\eta)^{2}\right\} \hbar \omega,
\end{aligned}
$$

where $\alpha$ indicates the fine-structure constant, $I$ the beam current, $e$ the charge of the beam particle, $\gamma$ the Lorentz factor, $\omega$ the angular frequency, $\omega_{c}$ the critical angular frequency, $\phi$ the bending angle, $\psi$ the angle to describe the deviation from the bending plane, $\xi \equiv \gamma \psi, \eta \equiv$ $(1 / 2)\left(\omega / \omega_{c}\right)\left(1+\xi^{2}\right)^{3 / 2}, \hbar$ Planck constant (reduced), and $K_{2 / 3}$ and $K_{1 / 3}$ are modified Bessel functions. Figure 3 explains the two variables $\phi$ and $\psi$. We regard $\Delta \phi$ as being an infinitesimal quantity, and the integral form for formula (7) is approximated by the following three-dimensional integration:

$$
\begin{aligned}
W= & \frac{3 \alpha}{4 \pi^{2}} \frac{I}{e} \gamma^{2} \iiint d \omega d s d \psi \frac{1}{\omega}\left(\frac{\omega}{\omega_{c}}\right)^{2}\left(1+\xi^{2}\right)^{2} \\
& \times\left\{K_{2 / 3}(\eta)^{2}+\frac{\xi^{2}}{1+\xi^{2}} K_{1 / 3}(\eta)^{2}\right\} \frac{\hbar \omega}{\rho(s)},
\end{aligned}
$$

where the $s$ axis is taken to be along the beam orbit, and $\rho(s)$ is the radius of curvature at $s$. A wattage distribution in a plane can be obtained by taking the mapping of $(s, \psi) \rightarrow(X, Y)$, where $(X, Y)$ is a point of intersection of the plane and the line with the angle $\psi$ with respect to the tangent of the orbit at $s$. Integration on $s$ and $\psi$

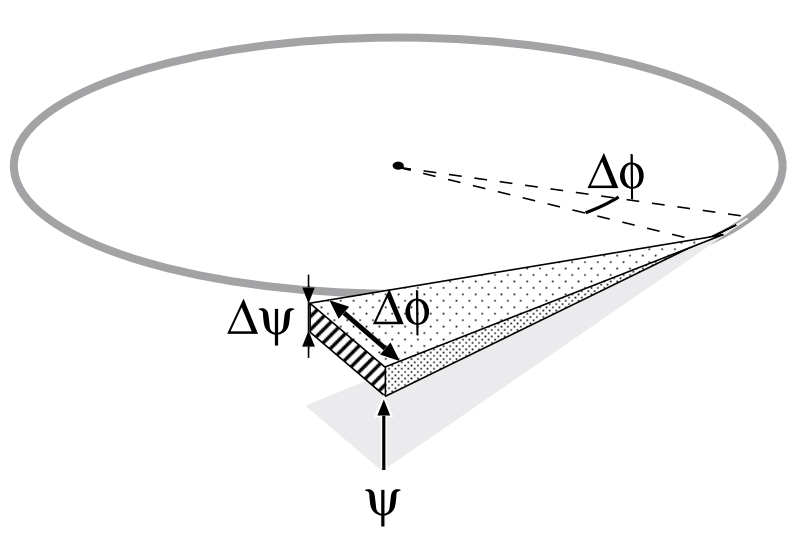

FIG. 3. Integration variables $\phi$ and $\psi$ used in the wattagecalculation formula (7). The circle indicates a circulating beam.

corresponds to surface integration of the Poynting vector in the plane. We perform numerical integrations of formula (8) using the Monte Carlo (MC) integration program BASES [7].

The implementation of the wattage calculation in our program was checked by comparing with the numerical calculation using SAD [8]. In the SAD calculation, the exact formula in electrodynamics [9] is used.

It is important to consider any beam-size effects in the SR simulation, especially for the SR from QUADs. The effects are included by adding four additional integration variables $\left(X, X^{\prime}, Y, Y^{\prime}\right)$, which indicate a phase-space point of the orbit at the IP. MC integration is performed together with the following Gaussian weight function to describe the beam profile:

$$
\frac{1}{(\sqrt{2 \pi})^{4} \sigma_{X} \sigma_{X^{\prime}} \sigma_{Y} \sigma_{Y^{\prime}}} \exp \left\{-\frac{\left(X-X_{\mathrm{fit}}\right)^{2}}{2 \sigma_{X}^{2}}\right\} \exp \left\{-\frac{\left(X^{\prime}-X_{\mathrm{fit}}^{\prime}\right)^{2}}{2 \sigma_{X^{\prime}}^{2}}\right\} \exp \left\{-\frac{\left(Y-Y_{\mathrm{fit}}\right)^{2}}{2 \sigma_{Y}^{2}}\right\} \exp \left\{-\frac{\left(Y^{\prime}-Y_{\mathrm{fit}}^{\prime}\right)^{2}}{2 \sigma_{Y^{\prime}}^{2}}\right\},
$$

where $\left(X_{\mathrm{fit}}, X_{\mathrm{fit}}^{\prime}, Y_{\mathrm{fit}}, Y_{\mathrm{fit}}^{\prime}\right)$ indicates the phase-space point of the fitted orbit at the IP, $\sigma_{X}\left(\sigma_{Y}\right)$ is the horizontal (vertical) beam size, and $\sigma_{X^{\prime}}$ and $\sigma_{Y^{\prime}}$ indicate the beam size in $X^{\prime}-Y^{\prime}$ space. 
The beam sizes are calculated according to the following formulas:

$$
\begin{gathered}
\sigma_{X}=\sqrt{\epsilon_{X} \beta_{X}^{*}}, \\
\sigma_{X^{\prime}}=\sqrt{\epsilon_{X} / \beta_{X}^{*}}, \\
\sigma_{Y}=\sqrt{\epsilon_{Y} \beta_{Y}^{*}}, \\
\sigma_{Y^{\prime}}=\sqrt{\epsilon_{Y} / \beta_{Y}^{*}},
\end{gathered}
$$

assuming $\alpha^{*}=0$, where $\alpha^{*}$ is one of the Courant-Snyder parameters at the IP in the notation $\gamma x^{2}+2 \alpha x x^{\prime}+$ $\beta x^{\prime 2}=\epsilon$. In the above formulas, $\epsilon_{X}\left(\epsilon_{Y}\right)$ indicates the horizontal (vertical) emittance, and $\beta_{X}^{*}\left(\beta_{Y}^{*}\right)$ is the horizontal (vertical) beta function at the IP. The orbits in the relevant magnets are calculated using the inverse transfer matrices from the IP. This implementation in our program has been checked by comparing the results with no beam size and with a beam size in the asymptotic behavior: $\epsilon_{X} \rightarrow 0$ and $\epsilon_{Y} \rightarrow 0$.

In Fig. 4, an example of the wattage distributions at the IP is shown in the plane perpendicular to the beam axis. The SR in this example was a dominant source in the SVD gain-drop accident.

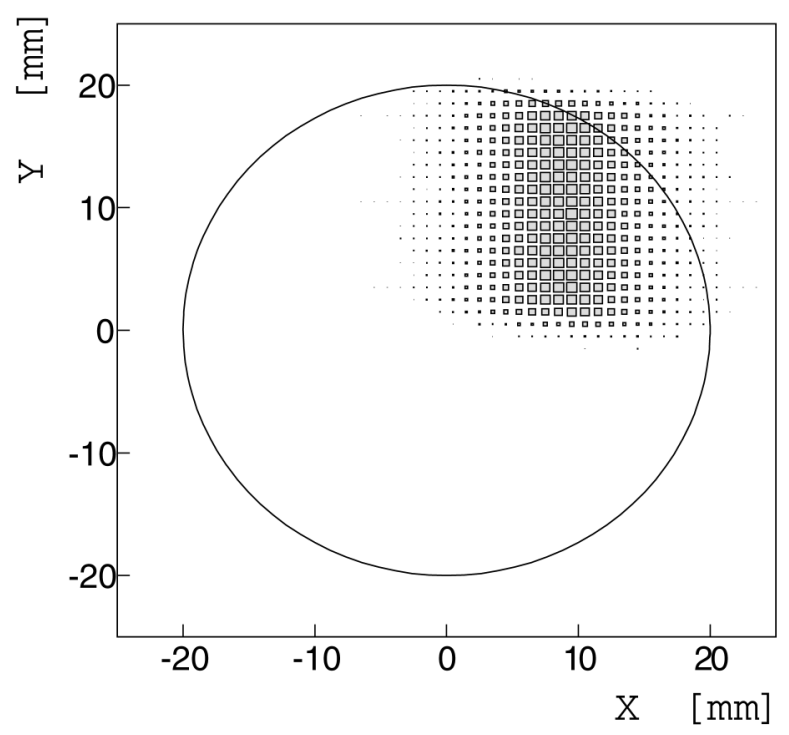

FIG. 4. Example of the wattage distributions at the IP in the plane perpendicular to the beam axis. The $X$ axis is taken to be in the horizontal direction, pointing to the outside of the KEKB ring, and $Y$ in the vertical direction. The SR comes from the vertical BEND located about $25 \mathrm{~m}$ upstream from the IP. The circle in the figure corresponds to the inner surface of the IP chamber.

\section{BENCHMARK TEST}

In order to demonstrate the validity of our method, we adopt the SVD gain-drop accident as a benchmark. For radiation-dose calculations, we made a detector simulation based on EGS4 [10], which was improved for lowenergy photons [11-15]. In this detector simulation, the exact geometry and materials of the relevant SVD components have been installed according to engineering drawings. SR photons, generated with the generalpurpose event generator SPRING [7], are fed into the detector simulation, and energy deposits in the SVD are calculated. Photons are simulated down to $1 \mathrm{keV}$, and electrons to $20 \mathrm{keV}$ kinematic energy. In order to transfer the radiation dose into the gain-drop, we use the result on the irradiation test to investigate the radiation hardness of the SVD preamplifiers [16].

We make two different sets of calculations for a more careful demonstration. One of them is on the radiation dose before starting the gain-drop (period I), and the other is after the start (period II), as shown in Fig. 5. During each of the two periods, the beam orbit was almost unchanged. Table I gives the results of a radiation-dose calculation for period I. Obtaining information on the time of the period (4641 min), and on the average beam current $(4.6 \mathrm{~mA})$, the gain-drop is estimated to be around or less than $1 \%$, which is consistent with the measurements ( $\lesssim 1 \%$ ). After starting the gaindrop, the estimated doses are much higher in period II than those in period I, as shown in Table II, where the time of the period is $8071 \mathrm{~min}$, and the average beam

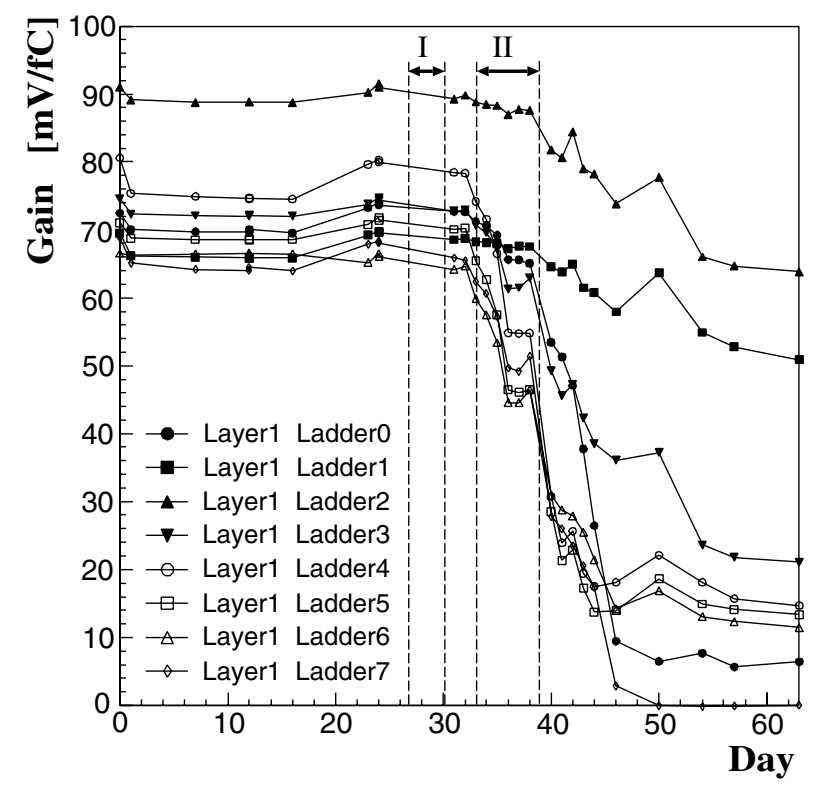

FIG. 5. SVD gain as a function of time around the SVD gaindrop accident, together with the two periods used in the benchmark test. The ladder numbers in this figure correspond to the position numbers in Tables I and II, and Fig. 7. The gain-drop plots are extracted from [17]. 
TABLE I. Results of a radiation-dose calculation based on our method for the period before starting the gain-drop (period I). The position numbers correspond to the azimuthal coordinate with respect to the beam axis, and the numbers $2,4,6,0$ indicate the top, inside, bottom, and outside of the KEKB ring, respectively. The errors from the MC statistics are shown beside the dose values.

\begin{tabular}{ccccccccc}
\hline \hline Position number & 1 & 2 & 3 & 4 & 5 & 6 & 7 & 0 \\
\hline Dose [rad min ${ }^{-1} \mathrm{~A}^{-1}$ ] & $2.1 \pm 0.9$ & $1.2 \pm 0.7$ & $23 \pm 3$ & $41 \pm 4$ & $33 \pm 4$ & $33 \pm 4$ & $25 \pm 3$ & $3.5 \pm 1.2$ \\
Integrated dose [krad] & 0.06 & 0.04 & 0.53 & 0.93 & 0.75 & 0.74 & 0.57 & 0.10 \\
Estimated gain-drop [\%] & 0.02 & 0.01 & 0.17 & 0.30 & 0.24 & 0.23 & 0.18 & 0.03 \\
\hline \hline
\end{tabular}

TABLE II. Results of a radiation-dose calculation for the period after starting the gain-drop (period II).

\begin{tabular}{ccccccccc}
\hline \hline Position number & 1 & 2 & 3 & 4 & 5 & 6 & 7 & 0 \\
\hline Dose $\left[10^{2} \mathrm{rad} \mathrm{min}^{-1} \mathrm{~A}^{-1}\right]$ & $1.5 \pm 0.4$ & $1.7 \pm 0.5$ & $10 \pm 1$ & $17 \pm 1$ & $24 \pm 2$ & $19 \pm 1$ & $19 \pm 1$ & $7.2 \pm 0.9$ \\
Integrated dose $[\mathrm{krad}]$ & 8.0 & 9.2 & 55 & 92 & 131 & 102 & 102 & 39 \\
Estimated gain-drop [\%] & 2.6 & 2.9 & 17.6 & 29.4 & 41.9 & 32.6 & 32.6 & 12.5 \\
\hline \hline
\end{tabular}
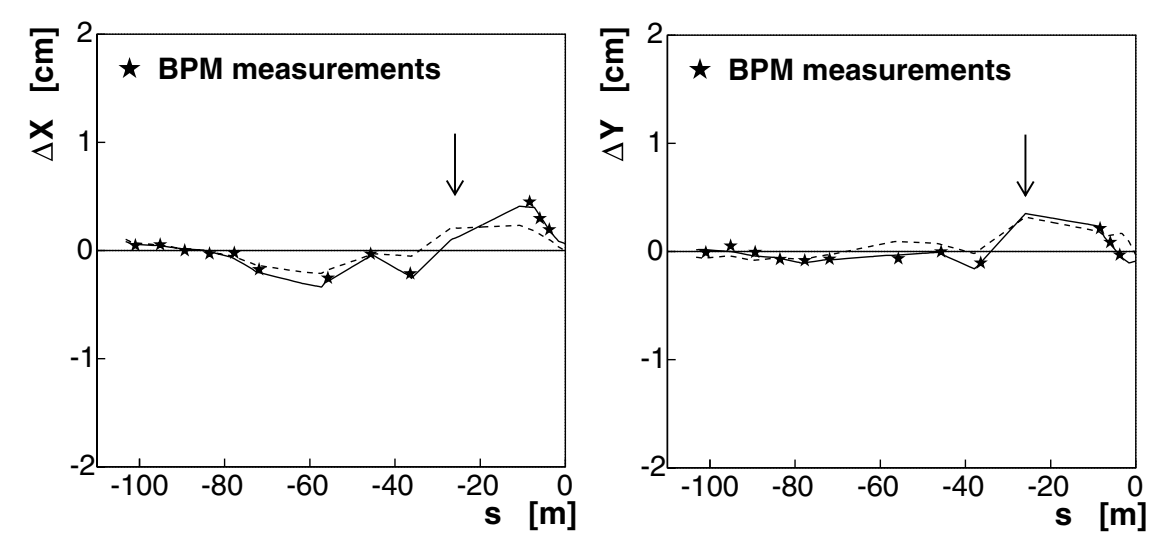

FIG. 6. Fitted beam orbits around the SVD gain-drop accident. The dotted line indicates the orbit in period I and the solid line in period II. The vertical arrow points to the BEND which made a dominant contribution to the SVD gain-drop in period II.

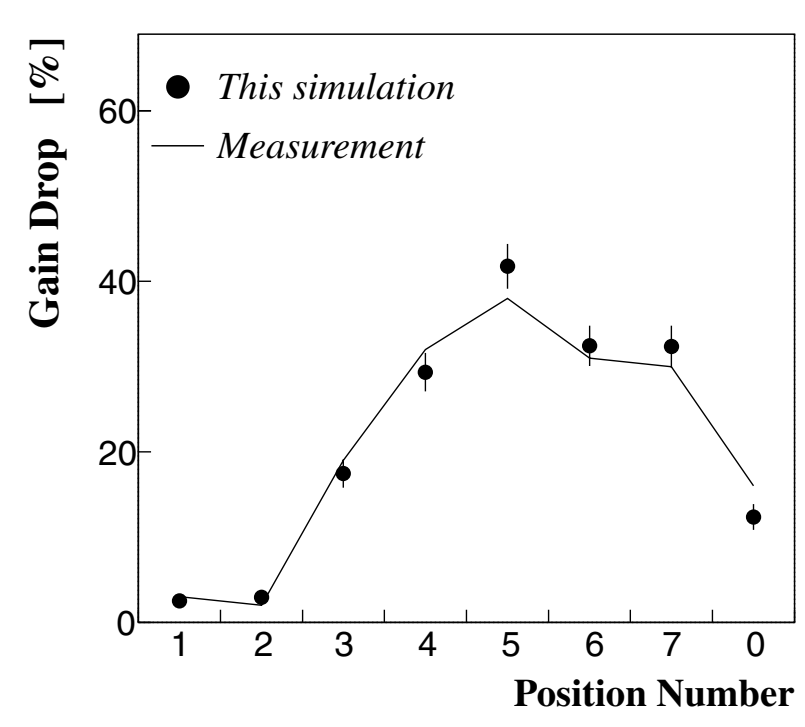

FIG. 7. Comparison between the estimated and measured gain-drop values for period II. current is $6.7 \mathrm{~mA}$. What changed significantly are the kick angle of the vertical BEND, which made a dominant contribution to the radiation dose in period II, and the orbit around the BEND, as shown in Fig. 6. The bending angle changed from 0.53 mrad with $1.7 \mathrm{keV}$ critical energy $\left(E_{\text {crit }}\right)$ to $0.66 \mathrm{mrad}$ with $E_{\text {crit }}=2.2 \mathrm{keV}$ in the same direction. Figure 7 shows a comparison between the estimated and measured gain-drop values. We have obtained good agreements not only on the absolute size, but also for the azimuthal distribution.

\section{CONCLUSIONS AND FUTURE PROSPECTS}

We have developed a new method to calculate SR wattages, or radiation doses, based on a real beam orbit with reasonable offset corrections. Here, the orbit is obtained by fitting BPM measurements. SR wattages are calculated based on the fitted orbit with the MC integration of the analytical formula. We have coded a dedicated computer program, where the correctness of the orbit and 
wattage calculations in our program has been confirmed by comparing with the results using SAD. Finally, we have successfully reproduced the SVD gain-drop accident quantitatively, so that the practicability of our method has been established.

This method can be applied to the construction of an on-line alarm system for SR background, leading to prior notice for detector damage and possibly more flexibility in accelerator operation. With this technique, a warning can be issued when the orbit is approaching dangerous areas, and furthermore the expected amount of SR background can be estimated before making certain changes in the orbit.

We have made a tentative version of the on-line program for KEKB operation. It takes about $20 \mathrm{~s}$ per cycle (getting the magnet information and BPM measurements, fitting the orbit, and MC integration of SR wattages from all the magnets in the HER straight section near the IP) using the workstation with a $500 \mathrm{MHz}$ CPU. If we use a recent faster computer, the turnaround time could be comparable to the time of BPM measurements (several seconds).

\section{ACKNOWLEDGMENTS}

We are grateful to Y. Funakoshi, S. Kamada, K. Kanazawa, M. Kikuchi, H. Koiso, M. Masuzawa, T. Nakamura, K. Oide, R. Sugahara, N. Yamamoto, J. Haba, S. Stanic, S. Swain, K. Trabelsi, T. Tsuboyama, S. Uno, M. Yokoyama, Y. Namito, and Y. Yamada for the fruitful comments and discussions.

\section{APPENDIX: TRANSFER MATRICES}

The exact forms of the transfer matrices used in the orbit calculation are shown in this appendix. Here, $L$ indicates the effective length of the component. $k$ is defined as $k=\sqrt{\left(B_{0} / b\right) /(B \rho)}$, where $B_{0}$ is the magnetic field strength at radius $b$, and $B \rho$ is the magnetic rigidity of the central reference trajectory. $\theta_{x}\left(\theta_{y}\right)$ is the horizontal (vertical) kick angle in the BEND. $\mathbf{R}_{\text {rot }}(\alpha)$ indicates the rotation matrix for the rotation angle, $\alpha$. The elements with a blank mean zero.

$$
\begin{aligned}
& \text { Orbit: } \mathbf{X}(s)=\left(\begin{array}{c}
X(s) \\
X^{\prime}(s) \\
Y(s) \\
Y^{\prime}(s) \\
\hline \mathbf{1}
\end{array}\right)
\end{aligned}
$$

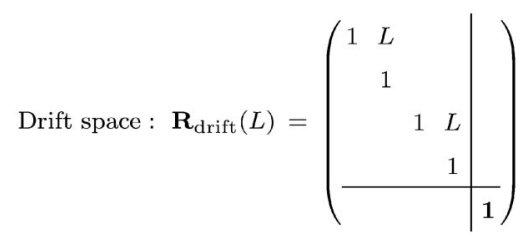

$$
\begin{aligned}
& \text { QUAD(horizontal focusing) : } \mathbf{R}_{\text {quad }}= \\
& \left(\begin{array}{cccc|c}
\cos (k L) & k^{-1} \sin (k L) & & & \\
-k \sin (k L) & \cos (k L) & & & \\
& & \cosh (k L) & k^{-1} \sinh _{1}(k L) & \\
& & k \sinh (k L) & \cosh (k L) & \\
\hline & & & 1
\end{array}\right) \\
& \mathrm{BEND}: \mathbf{R}_{\mathrm{band}}=\mathbf{R}_{\mathrm{drift}}\left(\frac{L}{2}\right) \times\left(\begin{array}{ccc|c}
1 & & & \\
& 1 & & \theta_{x} \\
& & 1 & \\
& & 1 & \theta_{y} \\
\hline & & & 1
\end{array}\right) \times \mathbf{R}_{\mathrm{drift}}\left(\frac{L}{2}\right)
\end{aligned}
$$

$$
\begin{aligned}
\text { Skew QUAD: } & \mathbf{R}_{\text {skew } Q}= \\
& \mathbf{R}_{\text {rot }}\left(-45^{\circ}\right) \times \mathbf{R}_{\text {quad }} \\
& \times \mathbf{R}_{\text {rot }}\left(+45^{\circ}\right) .
\end{aligned}
$$

[1] KEK Report No. 95-7, edited by N. Toge, 1995.

[2] Belle SVD Group, G. Alimonti et al., Nucl. Instrum. Methods Phys. Res., Sect. A 453, 71 (2000).

[3] SAD is a computer program for accelerator designs; http://acc-physics.kek.jp/SAD/sad.html.

[4] F. James, computer code MINUIT, version 94.1, 1994 (CERN Program Library Long Writeup D506).

[5] F. James and M. Roos, Comput. Phys. Commun. 10, 343 (1975).

[6] J. S. Schwinger, Phys. Rev. 75, 1912 (1949).

[7] S. Kawabata, Comput. Phys. Commun. 88, 309 (1995).

[8] Typically, it takes 10 hours to obtain a wattage distribution per a magnet using SAD; while using our program, shorter than $1 \mathrm{~s}$.

[9] SAD can calculate electric field produced by a point charge according to the Feynman- Heaviside formula which is derived from Maxwell's equations without any approximation.

[10] W. R. Nelson, H. Hirayama, and D.W. O. Rogers, SLAC Report No. SLAC-0265, 1985.

[11] Y. Namito, S. Ban, and H. Hirayama, Nucl. Instrum. Methods Phys. Res., Sect. A 349, 489 (1994).

[12] Y. Namito, H. Hirayama, and S. Ban, in Proceedings of the International Workshop on EGS4, Tsukuba, Japan, 1997 (KEK, Tsukuba, 1997).

[13] Y. Namito, H. Hirayama, and S. Ban, Radiat. Phys. Chem. 53, 283 (1998).

[14] H. Hirayama, Y. Namito, and S. Ban, KEK Internal Report No. 2000-3, 2000.

[15] Y. Namito and H. Hirayama, KEK Internal Report No. 2000-4, 2000.

[16] M. Yokoyama et al., IEEE Trans. Nucl. Sci. 48, 440 (2001).

[17] Belle SVD Group, Belle SVD2 Upgrade Technical Design Report (unpublished). 